\title{
Benefits of the Modified Bicaval Anastomosis Technique for Orthotopic Heart Transplantation From a Size-Mismatched Marginal Donor
}

\author{
Takashi Kakuta, MD; Satsuki Fukushima, MD, PhD; Yusuke Shimahara, MD, PhD; \\ Shin Yajima, MD, PhD; Naonori Kawamoto, MD; Naoki Tadokoro, MD; \\ Norihide Fukushima, MD, PhD; Soichiro Kitamura, MD, PhD; \\ Junjiro Kobayashi, MD, PhD; Tomoyuki Fujita, MD, PhD
}

\begin{abstract}
Background: Size-mismatched heart transplantation $(\mathrm{HTx})$ is associated with a risk of stenosis of the caval anastomosis site or low cardiac output syndrome. We developed a modified bicaval anastomosis technique (mBCAT) that achieved an adjustable caval anastomosis to compensate for size mismatch. This study was performed to validate the rationale of the mBCAT for size-mismatched HTx.

Methods and Results: This institutional consecutive series involved 106 patients who underwent HTx with the mBCAT during an 18 -year period. The cohort was divided into 3 groups according to the donor-to-recipient body weight ratio: $<0.8$, undersized group $(n=17)$; $0.8-1.3$, size-matched group $(n=68)$; and $>1.3$, oversized group $(n=21)$; outcomes were compared. The undersized, sizematched, and oversized groups showed no significant differences in the rate of mild or worse echocardiographic tricuspid regurgitation at 1 month [1 $(5.8 \%), 7(10.2 \%)$, and $1(4.8 \%)$, respectively; $P=0.87$ ] or the survival rate at 10 years $[100 \%, 93.9 \%$, and $100 \%$, respectively; $\mathrm{P}=0.25]$. The right heart catheter study revealed no pressure gradient across the orifices of both cavae in any patient. Additionally, the cardiac index immediately post-HTx was significantly low in the undersized group $(P=0.008)$, but was similar to the other groups at 6 months post-HTx $(\mathrm{P}=0.16)$.
\end{abstract}

Conclusions: The mBCAT prevented caval anastomosis-related complications in size-mismatched HTx and achieved excellent hemodynamics regardless of donor size.

Key Words: Heart transplantation; Modified bicaval anastomosis technique; Size-mismatched donor

$\mathbf{T}$ he critical donor shortage in Japan compared with other developed countries has promoted marginal donor usage in orthotopic heart transplantation (HTx) since the strict Japanese Organ Transplant Act became effective in 1999. Size-mismatched donors have thus been accepted, in addition to donors with previously established "marginal" factors such as advanced age, high inotrope infusion, low left ventricular ejection fraction (LVEF), a history of cardiopulmonary resuscitation, or a long ischemic time. ${ }^{1,2}$ In size-mismatched HTx, recipients with significantly undersized donors demonstrate higher mortality, ${ }^{3-10}$ and the International Society for Heart and Lung Transplantation guidelines caution the use of donors to within $30 \%$ of the recipient's body weight $(20 \%$ for a female donor and male recipient). ${ }^{11}$ "Undersize" HTx induces low cardiac output syndrome, inducing primary graft failure and impaired LV function or a long ischemic time in the donor heart. Additionally, caval anastomosis by the standard bicaval technique is technically demanding, especially in size-mismatched HTx, because of excessive tension or kinking of the superior vena cava (SVC), which can lead to caval anastomotic stenosis. ${ }^{12-14}$ In contrast, biatrial anastomosis has the advantage of easy, adjustable anastomosis, but its high incidence of both tricuspid regurgitation and pacemaker implantation are problematic. ${ }^{15,16}$

At the National Cerebral and Cardiovascular Research Center, Kitamura et al reported the modified bicaval anastomosis technique (mBCAT), in which the recipient's posterior right atrial wall is preserved to maintain continuity between the SVC and inferior vena cava (IVC), which avoids kinking and/or tension of the cavae and/or right atrium. ${ }^{17,18}$ Although this method achieves adjustable caval anastomosis to compensate for size mismatch, the post-

Received May 13, 2019; revised manuscript received October 20, 2019; accepted November 7, 2019; J-STAGE Advance Publication released online December 5, 2019 Time for primary review: 29 days

Department of Cardiac Surgery (T.K., S.F., Y.S., S.Y., N.K., N.T., S.K., J.K., T.F.), Department of Transplantation (N.F.), National Cerebral and Cardiovascular Research Center, Suita, Japan

Mailing address: Tomoyuki Fujita, MD, PhD, Chairman, Department of Cardiac Surgery, National Cerebral and Cardiovascular Research Center, 6-1 Kishibe-Shinmachi, Suita 564-8565, Japan. E-mail: tfujita@ncvc.go.jp

ISSN-1346-9843 All rights are reserved to the Japanese Circulation Society. For permissions, please e-mail: cj@j-circ.or.jp 


\begin{tabular}{|c|c|c|c|c|}
\hline & Undersize & Size-match & Oversize & $P$ value \\
\hline No. of patients (n) & 17 & 68 & 21 & \\
\hline \multicolumn{5}{|l|}{ Recipient factors } \\
\hline Age (years) & $43(36-52)$ & $40(30-50)$ & $31(25-46)$ & 0.22 \\
\hline Weight (kg) & $68.0(10.4)$ & $58.7(11.1)$ & $47.3(12.0)$ & $<0.001^{\#}$ \\
\hline Height (cm) & 169 (164-172) & $168(163-172)$ & $161(154-168)$ & $<0.001^{\#}$ \\
\hline $\mathrm{BSA}\left(\mathrm{m}^{2}\right)$ & $1.78(1.66-1.84)$ & $1.68(1.51-1.75)$ & $1.43(1.39-1.58)$ & $<0.001^{\#}$ \\
\hline $\mathrm{BMI}\left(\mathrm{kg} / \mathrm{m}^{2}\right)$ & $23.7(22.6-27.0)$ & $21.1(18.8-23.3)$ & $19.6(16.5-21.2)$ & $<0.001^{\#}$ \\
\hline Male:female (n) & $15: 2$ & $54: 14$ & $13: 8$ & 0.14 \\
\hline \multicolumn{5}{|l|}{ Diagnosis [n (\%)] } \\
\hline $\mathrm{DCM}$ & $11(64.0)$ & $49(72.0)$ & $13(61.9)$ & 0.59 \\
\hline $\mathrm{dHCM}$ & $1(5.8)$ & $5(7.3)$ & $5(23.8)$ & 0.09 \\
\hline $\mathrm{ICM}$ & $2(11.7)$ & $4(5.8)$ & $1(4.7)$ & 0.52 \\
\hline Other & $3(17.6)$ & $10(14.7)$ & $2(9.5)$ & 0.65 \\
\hline LVAD support & $17(100)$ & $64(94.1)$ & $20(95.2)$ & 1.00 \\
\hline LVAD supported time (days) & $1,071(227)$ & $1,002(318)$ & $842(373)$ & 0.08 \\
\hline Explanted heart weight (g) & 427 (365-467) & $372(315-445)$ & $366(323-455)$ & 0.34 \\
\hline \multicolumn{5}{|l|}{ Donor factors } \\
\hline Age (years) & $42.5(13.3)$ & $42.8(12.9)$ & $36.6(14.2)$ & 0.16 \\
\hline Weight (kg) & $52.0(7.4)$ & $60.1(11.7)$ & $70.3(18.3)$ & $<0.001^{\#}$ \\
\hline D/R BW ratio & $0.78(0.73-0.79)$ & $1.02(0.91-1.10)$ & $1.40(1.34-1.59)$ & $<0.001^{\#}$ \\
\hline Height (cm) & $160(157-167)$ & $166(157-170)$ & $173(165-175)$ & $0.02^{\#}$ \\
\hline $\mathrm{BSA}\left(\mathrm{m}^{2}\right)$ & $1.51(1.46-1.60)$ & $1.65(1.54-1.76)$ & $1.81(1.65-2.02)$ & $<0.001^{\#}$ \\
\hline $\mathrm{BMI}\left(\mathrm{kg} / \mathrm{m}^{2}\right)$ & $20.2(17.2-20.7)$ & $22.4(20.3-24.0)$ & $24.2(22.3-26.3)$ & $<0.001^{\#}$ \\
\hline Male:female (n) & $5: 12$ & 43:25 & $17: 4$ & $0.005^{\#}$ \\
\hline Sex-mismatch [n (\%)] & $10(58.8)$ & $17(25.0)$ & $3(14.2)$ & $0.008^{\#}$ \\
\hline Female to male $[\mathrm{n}(\%)]$ & $10(58.8)$ & $15(22.0)$ & $0(0)$ & $<0.001^{\#}$ \\
\hline Male to female [n (\%)] & $0(0)$ & $2(2.9)$ & $3(14.2)$ & 0.09 \\
\hline LVDd (mm) & $45(41-49)$ & $43(40-46)$ & $45(41-49)$ & 0.22 \\
\hline IVSd (mm) & $9(8-11)$ & $10(9-11)$ & $10(9-11)$ & 0.37 \\
\hline $\mathrm{PWd}(\mathrm{mm})$ & $9(8-10)$ & $10(9-11)$ & $11(12-12)$ & $0.01^{\#}$ \\
\hline LVMI $\left(g / m^{2}\right)$ & $76.9(13.9)$ & $85.4(21.9)$ & $100.7(44.7)$ & $0.001^{\#}$ \\
\hline $\operatorname{LVEF}(\%)$ & $61.5(9.5)$ & $62.4(8.5)$ & $64.2(11.1)$ & 0.65 \\
\hline High inotrope [n (\%)] & 0 & $10(14.7)$ & $5(23.8)$ & 0.09 \\
\hline Total ischemic time (min) & $173(153-197)$ & $195(177-217)$ & $205(180-215)$ & 0.10 \\
\hline
\end{tabular}

Categorical data are shown as number (percentage). Continuous data are shown as mean (standard deviation) or median (interquartile range). \#Indicate statistical significance. BSA, body surface area; BMI, body mass index; D/R BW ratio, donor/recipient body weight ratio; DCM, dilated cardiomyopathy; $\mathrm{dHCM}$, dilated phase of hypertrophic cardiomyopathy; HTx, heart transplantation; ICM, ischemic cardiomyopathy; IVSd, interventricular septal diameter; LVAD, left ventricular assist device; LVDd, left ventricular diastolic diameter; LVEF, left ventricular ejection fraction; LVMI, left ventricular mass index (donor LVM divided by the recipient's BSA); mBCAT, modified bicaval anastomosis technique; PWd, posterior wall diameter.

mBCAT hemodynamic changes after size-mismatched HTx are not fully understood. Therefore, this study was performed to validate the rationale of the $\mathrm{mBCAT}$ for sizemismatched HTx, with a particular focus on caval anastomotic site stenosis, tricuspid valve regurgitation (TR), and hemodynamic performance.

\section{Methods}

\section{Study Cohort and Data Collection}

The institutional surgical database contains a consecutive series of 111 patients who underwent orthotropic HTx in the National Cerebral and Cardiovascular Center Hospital from January 1999 to May 2018. Since the third such case, the mBCAT has been the standard technique for HTx; 5 patients who underwent HTx by the biatrial method $(n=4$; our first case and 3 patients with congenital anomalies) and conventional bicaval anastomosis $(\mathrm{n}=1$; our second case) were excluded from this study. The remaining 106 patients who underwent HTx with the mBCAT were enrolled. The patients' medical charts, surgical reports, and referral letters were reviewed to collect the data, which were further supplemented by telephone interviews of the patients under the care of distant physicians, including patients with major adverse cerebrovascular and cardiac events. As a result, complete follow-up of all the cohort was achieved. The median follow-up was 5.7 years (SD, 4.5 years; range, 1 month to 18 years). Data collection was performed from January 2018 to June 2018. All patients enrolled in this study provided informed consent, and the data collection, analysis, and reporting were approved by the institutional Review Board (reference no. M30-026). 


\begin{tabular}{|c|c|c|c|c|}
\hline & Undersize & Size-match & Oversize & $P$ value \\
\hline $\mathrm{Cl}$ at POD1 $\left(\mathrm{L} / \mathrm{min} / \mathrm{m}^{2}\right)$ & $2.6(0.4)$ & $3.0(0.6)$ & $3.1(0.5)$ & $0.01^{\#}$ \\
\hline ICU stay (days) & $4(3-5)$ & $4(3-6)$ & $5(3-6)$ & 0.47 \\
\hline Inotrope support (days) & $4(3-10)$ & $4(3-7)$ & $4(3-5)$ & 0.07 \\
\hline IABP support [n (\%)] & 0 & $7(10.2)$ & $1(4.7)$ & 0.66 \\
\hline ECMO usage [n (\%)] & 0 & $1(1.4)$ & 0 & 1.00 \\
\hline
\end{tabular}

Categorical data are shown as number (percentage). Continuous data are shown as mean (standard deviation) or median (interquartile range). "Indicate statistical significance. ECMO, extra-corporeal membrane oxygenation; $\mathrm{Cl}$, cardiac index; ICU, intensive care unit; IABP, intra-aortic balloon pumping; POD, postoperative day.

\begin{tabular}{|c|c|c|c|c|}
\hline & Undersize & Size-match & Oversize & $P$ value \\
\hline \multicolumn{5}{|l|}{ Echocardiography } \\
\hline LVDd (mm) & $41(40-45)$ & $41(39-45)$ & $40(39-42)$ & 0.88 \\
\hline IVSd (mm) & $8(8-9)$ & $9(8-10)$ & $10(9-11)$ & $0.004^{\#}$ \\
\hline $\mathrm{PWd}(\mathrm{mm})$ & $9(8-10)$ & $9(8-10)$ & $10(9-11)$ & $0.04^{\#}$ \\
\hline LVMI $\left(\mathrm{g} / \mathrm{m}^{2}\right)$ & $65.1(13.7)$ & $74.9(17.5)$ & $87.0(19.1)$ & $<0.001^{\#}$ \\
\hline LVEF (\%) & $60(56-64)$ & $62(56-67)$ & $64(53-68)$ & 0.19 \\
\hline AR grade (grade $0-4$ ) & $0(0-0)$ & $0(0-0)$ & $0(0-0.5)$ & 0.10 \\
\hline MR grade (grade $0-4$ ) & $0.5(0.5-1.0)$ & $0.5(0-0.5)$ & $0.5(0-0.5)$ & 0.35 \\
\hline TR grade (grade 0-4) & $1.0(0.5-1.0)$ & $0.5(0.5-1.0)$ & $0.5(0.5-1.0)$ & 0.41 \\
\hline $\mathrm{TR} \geq 2^{\circ}[\mathrm{n}(\%)]$ & $1(5.8)$ & $7(10.2)$ & $1(4.8)$ & 0.87 \\
\hline \multicolumn{5}{|l|}{ Right heart catheterization } \\
\hline Heart rate (beats/min) & $87.8(8.3)$ & $88.4(10.5)$ & $90.2(10.3)$ & 0.29 \\
\hline $\mathrm{Cl}\left(\mathrm{L} / \mathrm{min} / \mathrm{m}^{2}\right)$ & $3.0(0.4)$ & $3.4(0.7)$ & $3.6(0.7)$ & $0.008^{\#}$ \\
\hline PAWP (mmHg) & $8(7-10)$ & $8(6-12)$ & $8(5-10)$ & 0.77 \\
\hline Mean PAP $(\mathrm{mmHg})$ & $15(13-17)$ & $14(12-18)$ & $15(12-17)$ & 0.58 \\
\hline RAP $(\mathrm{mmHg})$ & $4(3-8)$ & $4(2-6)$ & $4(2-4)$ & 0.16 \\
\hline RAP/PAWP ratio & $0.50(0.37-0.82)$ & $0.50(0.38-0.67)$ & $0.44(0.38-0.58)$ & 0.21 \\
\hline RVSWI (g.m/m²/beat) & $4.7(3.9-5.4)$ & $5.0(3.8-6.4)$ & $6.5(5.0-7.7)$ & $0.01^{\#}$ \\
\hline PG between SVC or IVC and RA & \multicolumn{3}{|c|}{ No measureable PG in all patients } & 1.00 \\
\hline
\end{tabular}

Categorical data are shown as number (percentage). Continuous data are shown as mean (standard deviation) or median (interquartile range). "Indicate statistical significance. AR, aortic regurgitation; IVC, inferior vena cava; LVMI, left ventricular mass index; MR, mitral regurgitation; PAWP, pulmonary artery wedge pressure; PAP, pulmonary artery pressure; PG, pressure gradient; RA, right atrium; RAP, right atrial pressure; RVSWI, right ventricular stroke work index; SVC, superior vena cava; TR, tricuspid regurgitation. Other abbreviations as in Tables 1,2.

\section{Grouping of the Study Cohort}

The study cohort was grouped according to the transplant donor-to-recipient body weight ratio (D/R) as follows. The undersized group comprised 17 patients with a $\mathrm{D} / \mathrm{R}<0.8$, the size-matched group comprised 68 patients with a $D / R$ $0.8-1.3$, and the oversized group comprised 21 patients with a $D / R>1.3$. This grouping was based on the International Society of Heart and Lung Transplantation (ISHLT) guidelines, which caution the use of female donors to within $20 \%$ of the male recipient's body weight. Although the undersized group had a larger recipient body surface area (BSA) than the other groups, there was no significant difference in most of the recipient background variables among the 3 groups (Table 1). In addition, there were no significant differences in the characteristics of the donors among the 3 groups, including donor heart function, inotrope dose prior to organ procurement, or ischemic time (Table 1). Celsior solution was used for both cardioplegic arrest and immersion in the last 102 patients, whereas St. Thomas' Hospital solution was used in the initial 4 patients.

\section{Surgical Indication and Procedure of mBCAT}

The indications for HTx were discussed among the institutional heart team and the National Board of HTx based on clinical and social views. The patients were then registered as transplant candidates in the Japan Organ Transplant Network, which is a government-based sole organ transplant network in Japan. The recipient surgery was performed under general anesthesia with right heart catheter monitoring using a Swan-Ganz catheter in all patients. Briefly, the recipient's posterior right atrial wall is left undivided as a cuff bridging the SVC and IVC to maintain the anatomic orientation without traction, kinking, or distortion of the venae cavae. By expanding the suture line into the preserved posterior wall of the right atrium, anastomosis with a large donor heart can be accomplished. Additionally, the distance between the SVC and IVC can be adjusted in a small donor heart.

\section{Postoperative Immunosuppressive Treatment}

The initial immunosuppressive regimen in all cases comprised a combination of calcineurin inhibitor (tacrolimus 


\begin{tabular}{|c|c|c|c|c|}
\hline & Undersize & Size-match & Oversize & $P$ value \\
\hline \multicolumn{5}{|c|}{ Echocardiography } \\
\hline \multicolumn{5}{|l|}{ LVDd (mm) } \\
\hline 1 month & $41(40-45)$ & $41(39-45)$ & $40(39-42)$ & 0.88 \\
\hline 6 month & $42(40-44)$ & $42(38-46)$ & $41(37-43)$ & 0.91 \\
\hline 12 months & $43(39-46)$ & $42(38-45)$ & $41(36-44)$ & 0.87 \\
\hline \multicolumn{5}{|c|}{ LVDd/BSA $\left(\mathrm{mm} / \mathrm{m}^{2}\right)$} \\
\hline 1 month & $24.0(23.1-25.2)$ & $25.2(23.4-26.7)$ & 27.5 (25.5-29.2) & $<0.001^{\#}$ \\
\hline 6 months & $24.8(23.4-25.8)$ & $25.5(23.8-27.9)$ & $26.6(25.4-28.6)$ & $0.009^{\#}$ \\
\hline 12 months & $24.0(22.5-26.3)$ & $25.8(24.2-27.0)$ & $27.1(25.5-28.6)$ & $0.003^{\#}$ \\
\hline \multicolumn{5}{|l|}{ IVSd (mm) } \\
\hline 1 month & $8(8-9)$ & $9(8-10)$ & $10(9-11)$ & $0.004^{\#}$ \\
\hline 6 month & $9(8-9)$ & $9(8-10)^{\dagger}$ & $9(8-10)^{\dagger}$ & 0.12 \\
\hline 12 months & $8(8-9)$ & $8(8-10)^{\dagger}$ & $9(8-9)^{\dagger}$ & 0.36 \\
\hline \multicolumn{5}{|l|}{ PWd (mm) } \\
\hline 1 month & $9(8-10)$ & $9(8-10)$ & $10(9-11)$ & $0.04^{\#}$ \\
\hline 6 month & $9(8-9)$ & $9(8-10)^{\dagger}$ & $9(9-10)^{\dagger}$ & 0.08 \\
\hline 12 months & $8(8-9)$ & $9(8-9)^{\dagger}$ & $9(8-9)^{\dagger}$ & 0.16 \\
\hline \multicolumn{5}{|l|}{ LVMI $\left(\mathrm{g} / \mathrm{m}^{2}\right)$} \\
\hline 1 month & $65.1(13.7)$ & $74.9(17.5)$ & $87.0(19.1)$ & $<0.001^{\#}$ \\
\hline 6 months & $64.6(9.2)$ & $71.7(19.6)$ & $75.7(14.6)^{\dagger}$ & $0.03^{\#}$ \\
\hline 12 months & $62.6(10.9)$ & $68.6(16.7)^{\dagger}$ & $71.3(16.3)^{\dagger}$ & 0.07 \\
\hline \multicolumn{5}{|l|}{ LVEF (\%) } \\
\hline 1 month & $60(56-64)$ & $62(56-67)$ & $64(53-68)$ & 0.19 \\
\hline 6 month & $60(56-64)$ & $62(57-68)$ & $62(58-66)$ & 0.16 \\
\hline 12 months & $62(55-67)$ & $59(53-64)$ & $62(59-68)$ & 0.12 \\
\hline \multicolumn{5}{|c|}{ Right heart catheterization } \\
\hline \multicolumn{5}{|l|}{$\mathrm{Cl}\left(\mathrm{L} / \mathrm{min} / \mathrm{m}^{2}\right)$} \\
\hline 1 month & $3.0(0.4)$ & $3.4(0.7)$ & $3.6(0.7)$ & $0.008^{\#}$ \\
\hline 6 months & $3.5(0.5)^{\dagger}$ & $3.5(0.7)^{\dagger}$ & $3.7(0.7)$ & 0.16 \\
\hline 12 months & $3.6(0.6)^{\dagger}$ & $3.6(0.6)^{\dagger}$ & $4.0(0.7)^{\star}$ & 0.06 \\
\hline \multicolumn{5}{|c|}{ RVSWI (g.m/m²/beat) } \\
\hline 1 month & $4.7(3.9-5.4)$ & $5.0(3.8-6.4)$ & $6.5(5.0-7.7)$ & $0.01^{\#}$ \\
\hline 6 months & $6.4(4.9-8.6)^{\dagger}$ & $5.6(4.7-7.3)$ & $6.2(5.5-7.8)$ & 0.47 \\
\hline 12 months & $6.2(5.6-7.0)^{\dagger}$ & $6.3(4.9-7.3)^{\dagger}$ & $6.1(5.4-6.9)$ & 0.56 \\
\hline \multicolumn{5}{|c|}{ Serum BNP $(\mathrm{pg} / \mathrm{mL})$} \\
\hline 1 month & $178.3(82.5)$ & $208.3(187.0)$ & $243.7(242.1)$ & 0.51 \\
\hline 6 months & $63.4(37.0)^{\dagger}$ & $83.9(60.8)^{\dagger}$ & $58.8(31.5)^{\dagger}$ & 0.67 \\
\hline 12 months & $64.0(46.0)^{\dagger}$ & $84.1(101.8)^{\dagger}$ & $38.3(26.6)^{\dagger, *}$ & 0.97 \\
\hline
\end{tabular}

${ }^{\dagger} \mathrm{P}<0.05$ in comparison with the value at 1 month after heart transplantation. ${ }^{*} \mathrm{P}<0.05$ in comparison with the value at 6 months after heart transplantation. Categorical data are shown as number (percentage). Continuous data are shown as mean (standard deviation) or median (interquartile range). \#Indicate statistical significance. BNP, B-type natriuretic peptide. Other abbreviations as in Tables 1-3.

has been the standard in recent years), antimetabolites (mycophenolate mofetil has been the standard in recent years), and corticosteroids. In patients with renal dysfunction, an antibody agent (basiliximab has been the standard in recent years) was used for induction therapy or sparing of calcineurin inhibitors. Everolimus was never used in the initial stage; it was only introduced as an alternative to mycophenolate mofetil in the event of complications such as graft coronary artery lesions, renal dysfunction, malignant tumors, or intolerance.

\section{Laboratory Data Collection and Analysis}

All cohorts were examined by standard transthoracic echo- cardiography at 1, 6, and 12 months after HTx. Standard data were referred from the official echocardiographic report. The LV mass indexed to the BSA was estimated by the LV cavity dimension and wall thickness in the enddiastolic phase. Valve regurgitation was classified as grade 0 (none), 1 (trivial), 2 (mild), 3 (moderate), or 4 (severe). All cohorts also underwent a right heart catheter study at 1,6 , and 12 months after HTx, and serial changes in the hemodynamic parameters were assessed from the official catheter study report. The hemodynamic parameters assessed in this study were the heart rate, cardiac index (CI), pulmonary artery wedge pressure (PAWP), pulmonary artery pressure (PAP), and right atrial pressure 
(RAP). The right ventricular stroke work index (RVSWI) was calculated using these values. The catheter data for the CI at 1 day after HTx were collected from the medical record in the intensive care unit (ICU), where right heart catheter monitoring was continued. The serum B-type natriuretic peptide (BNP) level was measured 1, 6, and 12 months after HTx.

\section{Statistical Analysis}

Categorical data in Tables $\mathbf{1} \mathbf{- 4}$ are shown as number (\%). Continuous data were checked for normal distributions using the Shapiro-Wilk test. Normally distributed continuous data are shown as mean (standard deviation), while non-normally distributed continuous data are shown as median (interquartile range). Fisher's exact test was used to compare the categorical data among the 3 groups. For continuous data, because recipient weight, donor weight, LV assist device support time, donor age, donor heart LVEF, and heart rate were normally distributed, these values were compared between the groups using one-way analysis of variance. The remaining factors were non-normally distributed (recipient age, D/R, BSA, body mass index (BMI), total ischemic time, ICU stay, inotrope support, aortic regurgitation grade, mitral regurgitation grade, TR grade and pressure gradient); therefore, Kruskal-Wallis analysis was used. The Jonckheere-Terpstra trend test was applied to evaluate the postoperative hemodynamic parameters among the 3 groups, such as CI, PAWP, PAP, RAP, RVSWI, LV diastolic diameter (LVDd), intraventricular septal diameter (IVSd), posterior wall diameter (PWd), LV mass index (LVMI), EF, and serum BNP level. Time-dependent changes within the same group were compared using the paired t-test for normally distributed values and the Wilcoxon signed rank test for non-normally distributed values. Pairwise deletion was used to handle missing data. Time-related changes in the survival rate and rate of freedom from permanent pacemaker implantation were analyzed by the Kaplan-Meier method and log-rank test. A P-value $<0.05$ was considered statistically significant. All statistical analyses were performed using R 3.4.3 (The R Foundation for Statistical Computing, Vienna, Austria).

\section{Results}

\section{Comparative In-Hospital Outcomes Regardless of Size Difference}

No 30-day or in-hospital deaths occurred after HTx in any of the 106 patients, and all patients were discharged home.
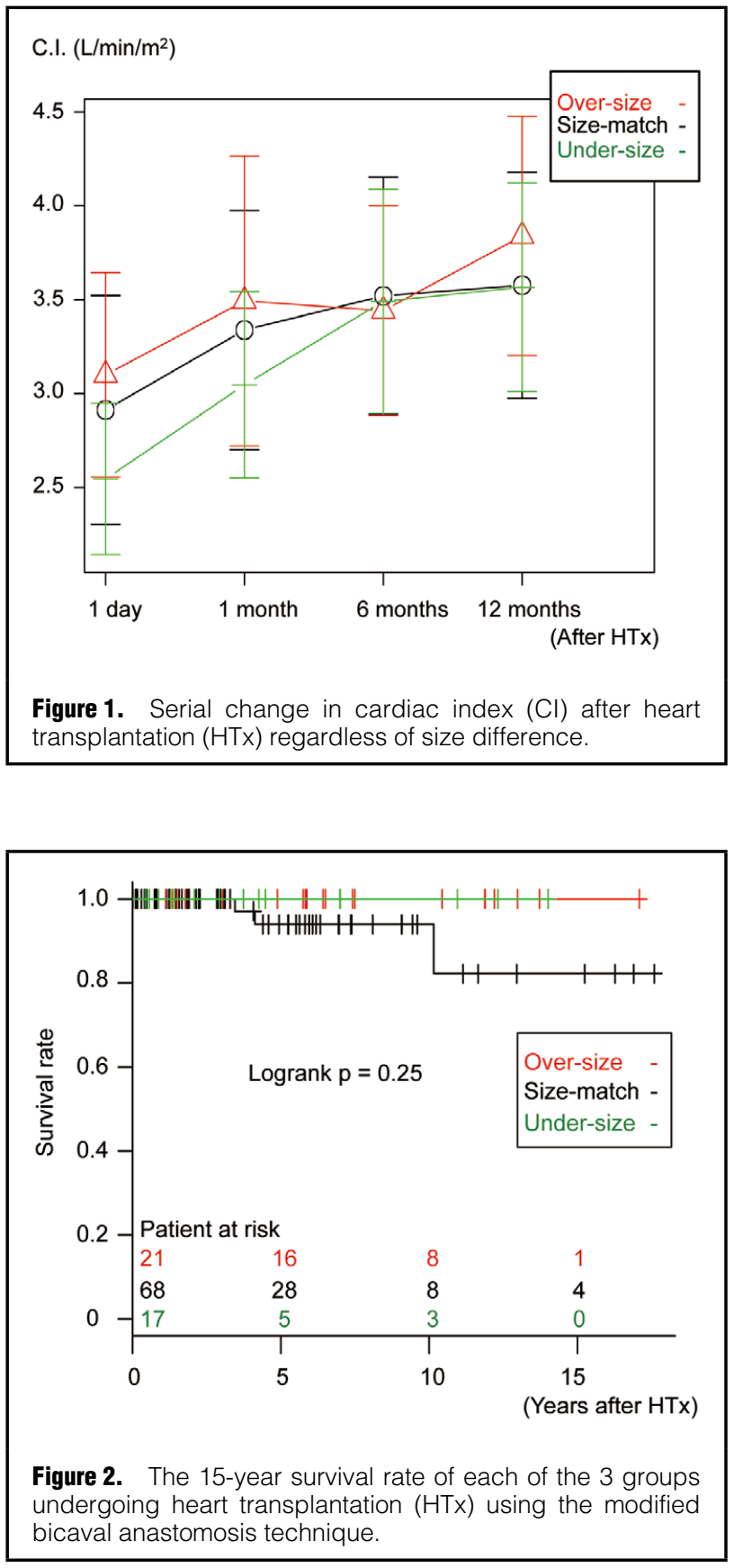

\begin{tabular}{|c|c|c|c|c|}
\hline & Undersize & Size-match & Oversize & $P$ value \\
\hline \multicolumn{5}{|c|}{$\beta$-blocker usage [n (\%)] } \\
\hline 1 month & 0 & $1(1.5)$ & 0 & 1.00 \\
\hline 6 months & 0 & $1(1.5)$ & 0 & 1.00 \\
\hline 12 months & 0 & $1(1.5)$ & 0 & 1.00 \\
\hline \multicolumn{5}{|c|}{ ACEI/ARB usage [n (\%)] } \\
\hline 1 month & $14(82.4)$ & $49(72.0)$ & $10(47.1)$ & 0.06 \\
\hline 6 months & $13(76.5)$ & $49(72.0)$ & $13(61.9)$ & 0.63 \\
\hline 12 months & $14(82.4)$ & $53(77.9)$ & $13(61.9)$ & 0.27 \\
\hline
\end{tabular}

Categorical data are shown as number (percentage). ACEI, angiotensin converting enzyme inhibitor; ARB, angiotensin II receptor blocker. 


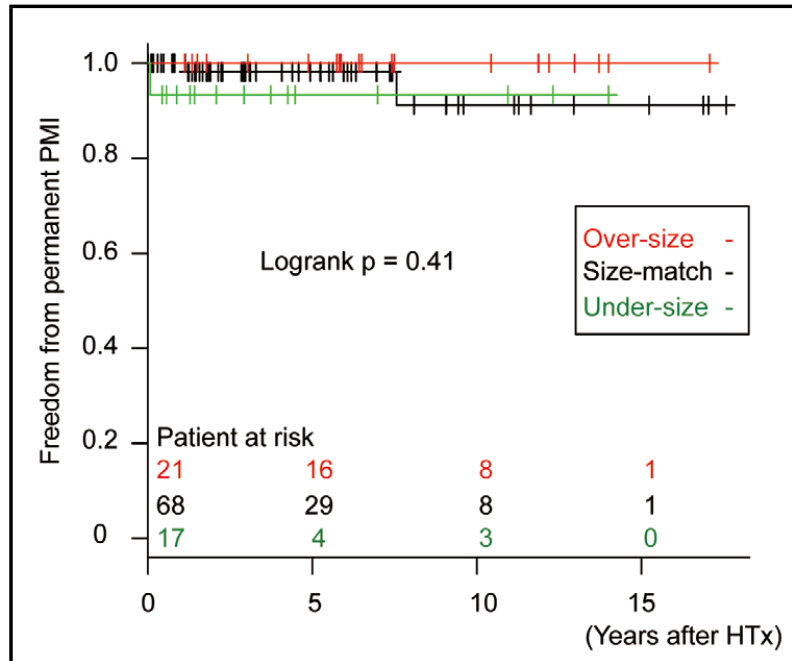

Figure 3. Rate of freedom from permanent pacemaker implantation (PMI) in each of the 3 groups. HTx, heart transplantation.

The CI calculated by Swan-Ganz catheter 1 day after HTx was $2.6(\mathrm{SD}, 0.4) \mathrm{L} / \mathrm{min} / \mathrm{m}^{2}$ in the undersized group, 3.0 (SD, 0.6) $\mathrm{L} / \mathrm{min} / \mathrm{m}^{2}$ in the size-matched group, and 3.1 $(\mathrm{SD}, 0.5) \mathrm{L} / \mathrm{min} / \mathrm{m}^{2}$ in the oversized group $(\mathrm{P}=0.01)$ (Table 2). The ICU stay was 4 (IQR, 3-5) days in the undersized group, 4 (IQR, 3-6) days in the size-matched group, and 5 (IQR, 3-6) days in the oversized group $(\mathrm{P}=0.46)$. The patients were postoperatively supported by inotropes for 4 (IQR, 3-10) days in the undersized group, 4 (IQR, 3-7) days in the size-matched group, and 4 (IQR, $3-5)$ days in the oversized group ( $\mathrm{P}=0.07)$. There was no significant difference in rate of postoperative circulatory support among the 3 groups (Table 2).

\section{Hemodynamic Differences at 1 Month Post-HTx by Size Difference}

Hemodynamic parameters at 1 month post-HTx were compared among the 3 groups using transthoracic echocardiography and the right heart catheter study. Although no significant differences in the LV dimension, LVEF, or degree of TR were found among the 3 groups, the IVSd, PWd and LVMI were significantly lower in the undersized group than in the other 2 groups (Table 3). Mild, moderate, or severe TR was present in $1(5.8 \%)$ patient of the undersized group, $7(10.2 \%)$ patients of the size-matched group, and $1(4.8 \%)$ patient of the oversized group $(\mathrm{P}=0.87)$. The right heart catheter study showed no pressure gradient across the orifices of the venae cavae in any patient. The PAWP, PAP, RAP, and RAP/PAWP ratio were not significantly different among the 3 groups, whereas the CI and RVSWI were significantly lower in the undersized group than in the other 2 groups (Table 3).

\section{Normalized Hemodynamics in the First 6 Months After Undersized HTX}

Serial changes in the CI, RVSWI, LVDd, LVMI, and serum BNP level in the first 12 months post-HTx were compared among the 3 groups. Trend testing showed that the CI and RVSWI at 1 month post-HTx were significantly smaller in the undersized group than in the other 2 groups.
These parameters in the undersized group gradually increased until 6 months post-HTx, showing no significant differences at 6 or 12 months post-HTx compared with the other 2 groups (Table 4). The serial changes in the CI from 1 day to 12 months post-HTx are presented in Figure 1. Trend testing showed that the LVMI at 1 month post-HTx was significantly smaller in the undersized group than in the other 2 groups; however, the LVMI in the undersized group did not change during the first 12 months after HTx, while the size-matched and oversized groups showed a reduction in LV size during the first 6 months after HTx. The LVDd/BSA parameter also showed a significant difference among the 3 groups at 1 month post-HTx and did not change in any group over the follow-up period. There was no significant difference between the 3 groups in the serum BNP levels at 1, 6, and 12 months after HTx.

\section{Comparative Long-Term Outcome Regardless of Size Difference}

Long-term outcomes after HTx were compared among the 3 groups: 3 patients in the size-matched group died of (1) pneumonia at 4 years post-HTx, (2) lymphoproliferative disorder at 4 years post-HTx, and (3) colon cancer at 10 years post-HTx. All surviving patients maintained New York Heart Association functional class I heart disease at the latest follow-up and had no episodes or signs suggestive of a hemodynamically significant host immune response. Rejection signs classified as greater than ISHLT grade $2 \mathrm{R}$ occurred in 1 patient $(5.9 \%)$ in the undersized group, in $5(7.4 \%)$ in the size-matched group, and in 1 $(4.8 \%)$ in the oversized group $(\mathrm{P}=0.27)$. There were no significant differences among the 3 groups in the medications administered in the first 12 months post-HTx (Table 5). The survival rate was $100 \%$ at 10 years in the undersized group; $100 \%$ at 1 year, $94 \%$ at 5 years, and $94 \%$ at 10 years in the size-matched group; and $100 \%$ at 10 years in the oversized group $(\mathrm{P}=0.25)$ (Figure 2).

During the follow-up, permanent pacemaker implantation was performed for sick sinus syndrome in 1 patient of the undersized group at 1 month post-HTx and for advanced atrioventricular block in 2 patients of the sizematched group at 1 and 8 years post-HTx, respectively. As a result, the respective 1-, 5-, and 10-year rates of freedom from permanent pacemaker implantation after HTx were $93 \%, 93 \%$, and $93 \%$ in the undersized group; $100 \%, 98 \%$, and $91 \%$ in the size-matched group; and $100 \%, 100 \%$, and $100 \%$ in the oversized group $(\mathrm{P}=0.41)$ (Figure 3).

\section{Discussion}

We performed HTx by mBCAT in 106 patients from 1999 to 2018 with no 30 -day or in-hospital deaths. The in-hospital postoperative course, including the ICU stay and inotrope support duration, was not affected by size mismatch between the donor and recipient. At 1 month postHTx, there were no differences in the LV dimensions, LVEF, RAP, PAP, or PAWP in relation to size mismatch, whereas the LVMI, CI, and RVSWI were significantly lower in the undersized HTx group. However, the undersize-associated hemodynamic differences at 1 month postHTx were fully diminished at 6 months post-HTx. Post-HTx survival was not affected by size mismatch, with $>90 \%$ survival at 10 years' post-HTx.

Undersized HTx carries a substantial risk of primary graft failure associated with the degree of undersize. 
Although the risk associated with undersized HTx is proposed to be high at $\mathrm{D} / \mathrm{R}<0.8,{ }^{11} 17$ patients $(16 \%)$ who underwent undersized HTx in the present study had such a ratio. We accepted these undersized donors with consideration of other risk factors, such as LV size and function prior to organ procurement or estimated ischemic time related to transportation. ${ }^{1,2}$ In fact, no donors had high inotrope support prior to organ procurement among those who underwent undersized HTx, and the ischemic time in undersized HTx was shorter than that for the other patients. As a result, some patients had clinically significant hemodynamic compromise after HTx despite the undersized transplant. Notably, the structural and functional characteristics of the patients who underwent undersized HTx had adjusted by 6 months post-HTx in the present study. Humoral factors of the recipient would enhance this remodeling of the donor heart. In addition, no patients in the present study who underwent undersized HTx showed excessive tension in the caval anastomosis or required graft interposition for caval anastomosis. The mBCAT contributed to adjustment of the caval anastomosis in the undersized transplant. Thorough assessment of the donor heart prior to organ procurement is critically important to ensure feasibility of undersized HTx from the viewpoint of the degree of undersizing, the donor heart's function, and the ischemic time.

In contrast, a particular concern of oversized HTx is kinking and/or distortion of the pathway from the vena cava to the right atrium, which could induce critical congestion of the upper and/or lower body and thus compromise systemic hemodynamics. The mBCAT leaves a strip of the posterior right atrial tissue to maintain continuity between the SVC and IVC, which enhances trimming of the oversized right atrial tissue of the donor heart to adjust the caval anastomosis. In fact, no patients in the present study showed hemodynamically significant obstruction in the caval-right atrial pathway, even among all 21 patients who underwent oversized HTx. Notably, the structural and functional characteristics were almost identical between the patients who underwent oversized and sizematched HTx in the present study, which indicates that hemodynamic adjustment is more promptly established in the oversized than undersized donor heart.

Although the present study defined size mismatch only by body weight in accordance with the current guidelines, 11 we consider that height might also influence the size mismatch. In fact, it has previously been demonstrated that the BMI, which is calculated using weight and height, is a factor defining size mismatch in HTx, and that undersized mismatch HTx is associated with death. ${ }^{3}$ Furthermore, an obese recipient overestimates the size mismatch. ${ }^{3}$ In contrast to the previous study in which more than $18 \%$ of patients had a BMI $>30,{ }^{3}$ the present cohort showed a consistent BMI, with only 2 patients $(1.9 \%)$ in the total cohort having a BMI $>30$. Height and BMI therefore showed a similar trend to weight in the present cohort.

Several studies have emphasized the increased accuracy of using cardiac size rather than body weight as an indicator of size mismatch, despite the ISHLT guidelines that advise the use of weight as an indicator of size mismatch. One previous study reported a relationship between total cardiac mass size and undersized mismatch HTx, ${ }^{5}$ while another study found that the difference in body weight explained only $17 \%$ of the variance in the LVDD. ${ }^{19}$ In the present study, there were significant differences in both of the corrected parameters, such as the LVMI and the LVDd/BSA, at 1 month and 6 months after HTx among the 3 groups, which suggests that the weight-undersized transplant represents both the heart muscle-undersized and the heart cavity-undersized transplant. Therefore, we consider it reasonable to assume that weight is accurately related to cardiac size as an indicator of size-mismatched HTx. However, weight may have a stronger connection to heart muscle size than to heart cavity size because in the present study the LVMI changed in the post weight-mismatched HTx, but not the LVDd or BSA.

Cardiac remodeling post-HTx is affected by multiple factors. Although the present study did not dissect the mechanisms of the remodeling, it has been investigated in previous studies. ${ }^{20,21}$ The onset of cardiac remodeling postHTx is affected by ischemia and denervation of the donor heart and the circulatory demands of the recipient body, which affect the donor heart in a multidirectional way via catecholamines, neurohormones, growth factors, and cytokines. Cross-talk between the donor heart and recipient body continues until completion of the remodeling of the donor heart.

In the present study, the LVMI did not change during the 12 months post-HTx in the undersized group, but reduced in the first 6 months post-HTx in the size-matched and oversized groups. In contrast, the CI and RVSWI increased in the first 6 months post-HTx in the undersized group, but did not change over time in the size-matched and oversized groups. This finding suggested that the RV may be more vulnerable to undergoing adjustments to suit the new body, thus inducing cardiac remodeling, than the $\mathrm{LV}$; however, this must be confirmed in further studies of the dynamic changes in the RV and the LV after HTx.

\section{Study Limitations}

The present study was primarily limited by its retrospective design. The indication for size-mismatched HTx was discussed by the heart team as the best clinical option for the individual patient without clear documentation of the inclusion or exclusion criteria.

In conclusion, the mBCAT prevented caval anastomosis-related complications in size-mismatched HTx and achieved excellent hemodynamics regardless of the donor size, possibly warranting further expansion of the indication for size-mismatched HTx.

\section{Acknowledgments}

We thank Kelly Zammit, BVSc, and Angela Morben, DVM, ELS, from Edanz Group (www.edanzediting.com/ac) for editing a draft of this manuscript.

\section{Disclosure Statement}

The authors have no conflicts of interest to disclose. No funding was obtained for this work.

\section{References}

1. Fujita T, Toda K, Yanase M, Seguchi O, Murata Y, IshibashiUeda H, et al. Risk factors for post-transplant low output syndrome. Eur J Cardiothorac Surg 2012; 42: 551-556.

2. Fukushima N, Ono M, Saito S, Saiki Y, Kubota S, Tanoue Y, et al. Japanese strategies to maximize heart and lung availabilities: Experience from 100 consecutive brain-dead donors. Transplant Proc 2013; 45: 2871-2874.

3. Bergenfeldt H, Stehlik J, Höglund P, Andersson B, Nilsson J. Donor-recipient size matching and mortality in heart transplantation: Influence of body mass index and gender. J Heart Lung 
Transplant 2017; 36: 940-947.

4. Ziaziaris W, Chew HC, Dhital K, Hayward C, Pleass H, Macdonald P. Size and gender matching in heart transplantation: Optimizing donor utilization in an era of changing donor and recipient characteristics. Curr Transplant Rep 2014; 1: $266-272$.

5. Reed RM, Netzer G, Hunsicker L, Mitchell BD, Rajagopal K, Scharf S, et al. Cardiac size and sex-matching in heart transplantation: Size matters in matters of sex and the heart. JACC Heart Fail 2014; 2: 73-83

6. Jayarajan SN, Taghavi S, Komaroff E, Mangi AA. Impact of low donor to recipient weight ratios on cardiac transplantation. J Thorac Cardiovasc Surg 2013; 146: 1538-1543.

7. Stehlik J, Feldman DS, Brown RN, VanBakel AB, Russel SD, Ewald GA, et al. Interactions among donor characteristics influence post-transplant survival: A multi-institutional analysis. $J$ Heart Lung Transplant 2010; 29: 291-298.

8. Weiss ES, Allen JG, Patel ND, Russell SD, Baumgartner WA, Shah AS, et al. The impact of donor-recipient sex matching on survival after orthotopic heart transplantation: Analysis of 18000 transplants in the modern era. Circ Heart Fail 2009; 2: 401-408.

9. Patel ND, Weiss ES, Nwakanma LU, Russell SD, Baumgartner WA, Shah AS, et al. Impact of donor-to-recipient weight ratio on survival after heart transplantation: Analysis of the United Network for Organ Sharing Database. Circulation 2008; 118: S83-S88.

10. Smits JM, Vanhaecke J, Haverich A, de Vries E, Smith M, Rutgrink E, et al. Three-year survival rates for all consecutive heart-only and lung-only transplants performed in Eurotransplant, 1997-1999. Clin Transpl 2003; 89-100.

11. Costanzo MR, Dipchand A, Starling R, Anderson A, Chan M, Desai S, et al. The International Society for Heart and Lung Transplantation guidelines for the care of heart transplant recipients. J Heart Lung Transplant 2010; 29: 914-956.

12. Jacob S, Sellke F. Is bicaval orthotopic heart transplantation superior to the biatrial technique? Interact Cardiovasc Thorac
Surg 2009; 9: 333-342.

13. Sze DY, Robbins RC, Semba CP, Razavi MK, Dake MD. Superior vena cava syndrome after heart transplantation: Percutaneous treatment of a complication of bicaval anastomoses. $J$ Thorac Cardiovasc Surg 1998; 116: 253-261.

14. Shah M, Anderson AS, Jayakar D, Jeevanandam V, Feldman T. Balloon-expandable stent placement for superior vena cava-right atrial stenosis after heart transplantation. J Heart Lung Transplant 2000; 19: 705-709.

15. Wartig M, Tesan S, Gäbel J, Jeppsson A, Selimovic N, Holmberg $\mathrm{E}$, et al. Tricuspid regurgitation influences outcome after heart transplantation. J Heart Lung Transplant 2014; 33: 829-835.

16. Davies RR, Russo MJ, Morgan JA, Sorabella RA, Naka Y, Chen JM. Standard versus bicaval techniques for orthotopic heart transplantation: An analysis of the United Network for Organ Sharing database. J Thorac Cardiovasc Surg 2010; 140: 700-708, 708.e701-702.

17. Kitamura S, Nakatani T, Kato T, Yanase M, Kobayashi J, Nakajima H, et al. Hemodynamic and echocardiographic evaluation of orthotopic heart transplantation with the modified bicaval anastomosis technique. Circ $J$ 2009; 73: 1235-1239.

18. Kitamura S, Nakatani T, Bando K, Sasako Y, Kobayashi J, Yagihara T. Modification of bicaval anastomosis technique for orthotopic heart transplantation. Ann Thorac Surg 2001; 72: $1405-1406$

19. Chan BB, Fleischer KJ, Bergin JD, Peyton VC, Flanagan TL, Kern JA, et al. Weight is not an accurate criterion for adult cardiac transplant size matching. Ann Thorac Surg 1991; 52: $1230-1235$.

20. Delmo Walter EM, Huebler M, Stamm C, Alexi-Meskishvili V, Weng Y, Berger F, et al. Adaptive growth and remodeling of transplanted hearts in children. Eur J Cardiothorac Surg 2011; 40: $1374-1382$.

21. Tang L, Du W, Delius RE, L'Ecuyer JL, Zilberman MV. Low donor-to-recipient weight ratio does not negatively impact survival of pediatric transplant patients. Pediatr Transplant 2010; 14: $741-745$. 